https://helda.helsinki.fi

\title{
Tracking self-citations in academic publishing
}

\section{Kacem, Ameni}

2020-05

Kacem , A , Flatt , J W \& Mayr , P 2020 , ' Tracking self-citations in academic publishing ' , pÿScientometrics , vol. 123 , no. 2 , pp. 11571165 . https://doi.org/10.1007/s11192-020-03413-9

http://hdl.handle.net/10138/317394

https://doi.org/10.1007/s11192-020-03413-9

cc_by

publishedVersion

Downloaded from Helda, University of Helsinki institutional repository.

This is an electronic reprint of the original article.

This reprint may differ from the original in pagination and typographic detail.

Please cite the original version. 


\title{
Tracking self-citations in academic publishing
}

\author{
Ameni Kacem ${ }^{1}$ (D) J Justin W. Flatt ${ }^{2,3}$ (D) Philipp Mayr ${ }^{1}$ (D)
}

Received: 26 February 2020 / Published online: 18 March 2020

(c) The Author(s) 2020

\begin{abstract}
Citation metrics have value because they aim to make scientific assessment a level playing field, but urgent transparency-based adjustments are necessary to ensure that measurements yield the most accurate picture of impact and excellence. One problematic area is the handling of self-citations, which are either excluded or inappropriately accounted for when using bibliometric indicators for research evaluation. Here, in favor of openly tracking self-citations we report on self-referencing behavior among various academic disciplines as captured by the curated Clarivate Analytics Web of Science database. Specifically, we examined the behavior of 385,616 authors grouped into 15 subject areas like Biology, Chemistry, Science and Technology, Engineering, and Physics. These authors have published 3,240,973 papers that have accumulated 90,806,462 citations, roughly five percent of which are self-citations. Up until now, very little is known about the buildup of self-citations at the author-level and in field-specific contexts. Our view is that hiding self-citation data is indefensible and needlessly confuses any attempts to understand the bibliometric impact of one's work. Instead we urge academics to embrace visibility of citation data in a community of peers, which relies on nuance and openness rather than curated scorekeeping.
\end{abstract}

\section{Introduction}

Metrics of productivity can be valuable in assisting evaluation, but to do this they must provide complete and accurate descriptions of citations (Cousijn et al. 2018). Currently this is not the case. Fixing the problem is deceptively simple for a variety of reasons, one being that there is no consensus on how to handle self-citation data. The ongoing debate is

Justin W. Flatt

justin.flatt@helsinki.fi

Philipp Mayr

philipp.mayr@gesis.org

Ameni Kacem

ameni.kacem@gmail.com

1 GESIS - Leibniz Institute for the Social Sciences, Unter Sachsenhausen 6-8, 50667 Cologne, Germany

2 Institute of Biotechnology, University of Helsinki, 00790 Helsinki, Finland

3 Department of Biosciences, University of Helsinki, 00790 Helsinki, Finland 
contentious and further complicated by the widespread use of the $h$-index for research evaluation, which as the dominating metric puts the emphasis squarely on citations to guide decision making (Hirsch 2005; Hicks et al. 2015). Without question, this creates a real career motivation to strategically use self-citation (Seeber et al. 2019), but this does not in any way diminish the value of self-cites that result from productive, sustained, leadingedge efforts (Cooke and Donaldson 2014). When used appropriately, self-cites are equally important as cites from the surrounding community, and without tracking them it is impossible to see how scholars build on their own work.

Despite this, many favor a curated form of the $h$-index as a response to the gaming problem. Curation involves hacking away at the citation data to neatly remove all occurrences of self-citation. While such treatment effectively silences direct attempts to boost citation scores, it does not prevent indirect manipulation and also produces undesired side effects. For example, curation ignores when authors use self-citation to attract cites from others, which is alarming given that each self-citation appears to increase the number of citations from others by about one after a year, and by about three after 5 years (Fowler and Aksnes 2007). Furthermore, curation unfairly punishes good citation practices, a particularly worrisome issue for those publishing novel ideas or results that challenge well-established dogma. In such cases, self-citation data can be critical as paper outputs may require a substantially longer period of time to attract the attention (i.e., citations) they ultimately deserve. Thus it is not good practice to hide self-citation data. The end result is a distorted record of progress and discovery.

The sensible alternative to curated scorekeeping would be to consider all citation data including self-citations. Towards this goal, we demonstrate an easy way to track selfcites without distorting other metrics, namely the $h$-index. The approach is not meant to criminalize self-referencing, nor do we intend to suggest a certain threshold of acceptable behavior like what Hirsch did when proposing the $h$-index (Hirsch 2005). Rather we see this as a tool to clarify how researchers build on their own ideas, as well as how self-citing contributes to the bibliometric impact of their own work. Furthermore, researchers are less likely to blatantly boost their own citation scores (Zhivotovsky and Krutovsky 2008; Bartneck and Kokkelmans 2011) while others are watching.

\section{Defining and tracking self-citation in academic publishing}

For author-level tracking, we define a self-citation as any instance where a given author cites their own articles. How we define self-citation differs from recent work done by Ioannidis et al. (2019) where they count a self-citation as any occasion where an author of a given article cites that article. Our reason for this is that we want to know how often specific authors self-cite, not how often an article gets cited by coauthors. In general, we believe that authors' citations should be sorted by source for clarification: self, nonself, coauthor, etc. and tracked separately. We focus here on self-citation data to show how the approach could work.

To study self-citation practices and regularities in field-specific contexts we analyzed citation data from the 2016 Web of Science database via the German Competence Centre for Bibliometrics. Authors with a unique identifier (either ORCID or ResearcherID) were classified into research areas based on the Web of Science categorization scheme. At the level of category, we found that self-citation accounts for only a small percentage of total citations (Fig. 1a), however, there is high variation in self-referencing patterns among 

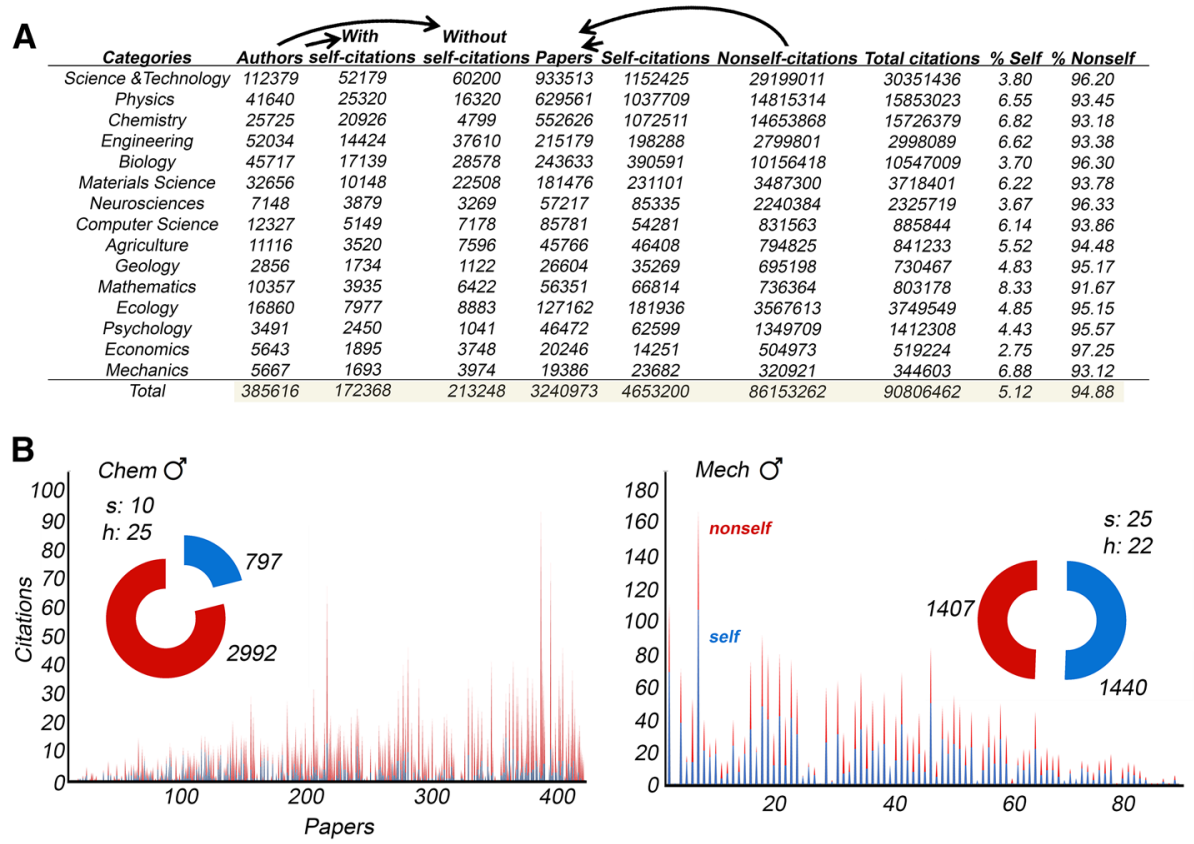

Fig. 1 Tracking authors' self-citation. a Authors were categorized into 15 subject areas and citations were sorted based on whether they were self or nonself in origin. The total amount of authors, papers, and citations analyzed is highlighted in brown. b Examples of author profiles where citations have been clarified to separate self-(blue) and nonself-citations (red). Papers are sorted from oldest to most recent. Gender (manually verified), category, $s$-index, and $h$-index (excluding self-citations) are reported. (Color figure online)

individual authors (Fig. 1b). This heterogeneity has implications for how we make generalizations regarding self-citation practices. In particular, it does not make sense to use summary statistics to capture average trends when averages would provide such a poor description of individual behavior. There is no metric substitute for expert peer review.

\section{Computing $s$ alongside $h$}

We recently proposed a self-citation index ( $s$-index), which is little more than a modified $h$-index.

A scientist has index $s$ if $s$ of his or her $N p$ papers have at least $s$ self-citations each and the other $(\mathrm{Np}-s)$ papers have $\leq s$ self-citations each (Flatt et al. 2017).

The rationale behind the $s$-index is that it brings urgently needed context (e.g., self-citation data) to the $h$-index without introducing distortions. This is especially important given that the $h$-index has become immovable for evaluation and comparison purposes. Towards implementation, we have computed $s$-index scores for all the authors in our study (Fig. 2a). From the reported scores, it can be seen that $98 \%(377,533)$ of authors achieve an $s$-index of 5 or less, $1.9 \%$ (7561) achieve a score between 6 and 10, and only $0.1 \%$ (522) exceed 10. 


\section{A}

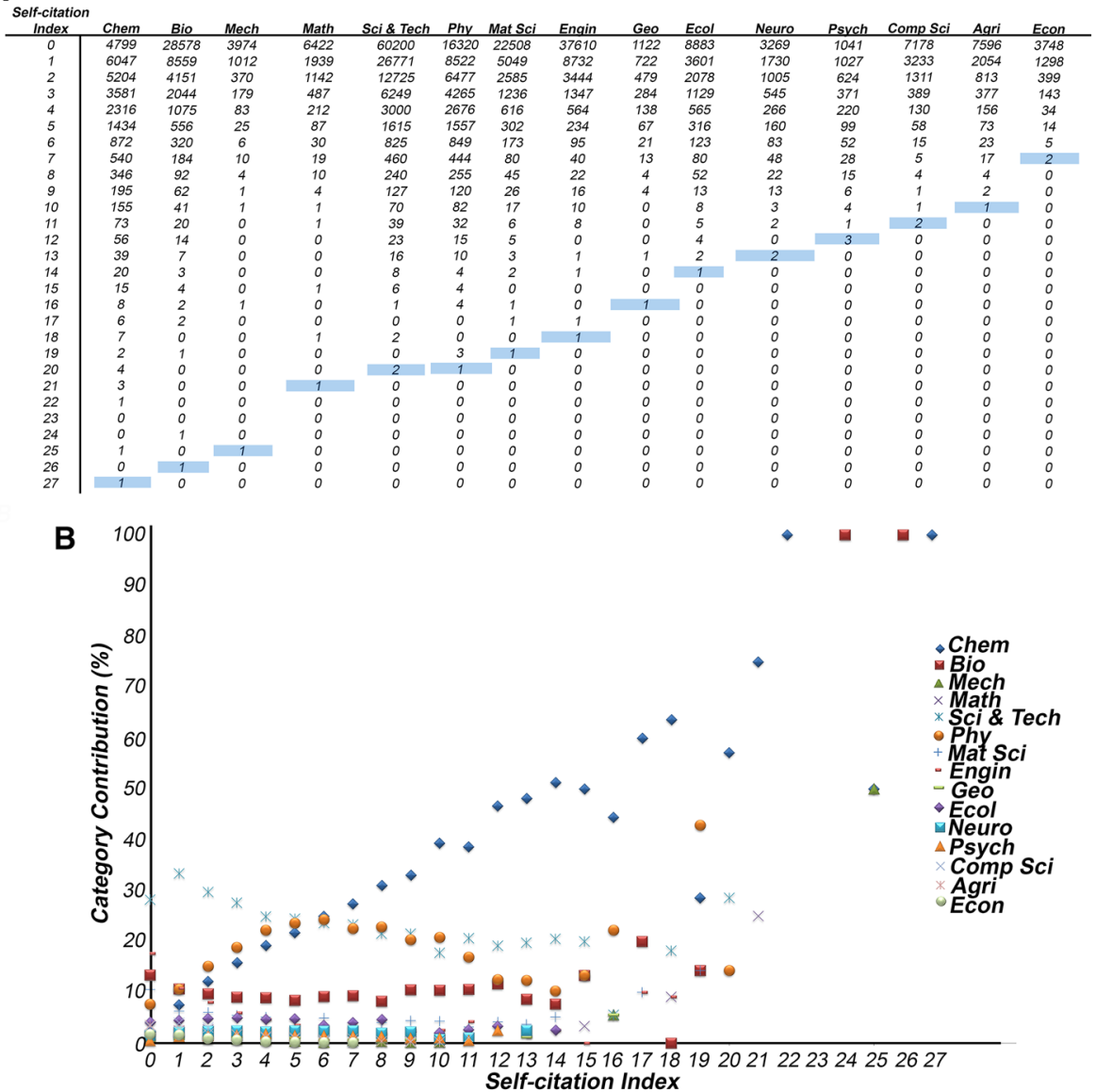

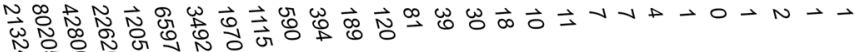
Number of Authors Per Score

Fig. $2 s$-index scores in field-specific contexts. a Table shows how authors are distributed according to $s$-index for the 15 categories considered in this study. Highest scores are highlighted in blue (e.g. Chem27, Ecol-14). b Graph depicts how the categories contribute to observed $s$ outcomes. For example, the category Chem is $100 \%$ responsible for the one author that achieves a score of 27 , whereas both Chem and Mech contain authors with an $s$ of 25 , and thus they each contribute $50 \%$ to the total (here, 2 authors achieve $s$ of 25). (Color figure online)

In terms of high scorers, the research categories most represented were Chemistry (236), Science and Technology (97), Physics (73), and Biology (55) (Fig. 2b).

We have also included information about the top $s$-index scorers for the different categories (Fig. 3). Each of the authors depicted is male, beyond the "early" phase of their careers, and productive in terms of paper outputs and citations. The key difference being the percentage of total citations that are self-citations, which varies from 3\% (Psychology) to $58 \%$ (Engineering). This difference only becomes apparent by looking at the 

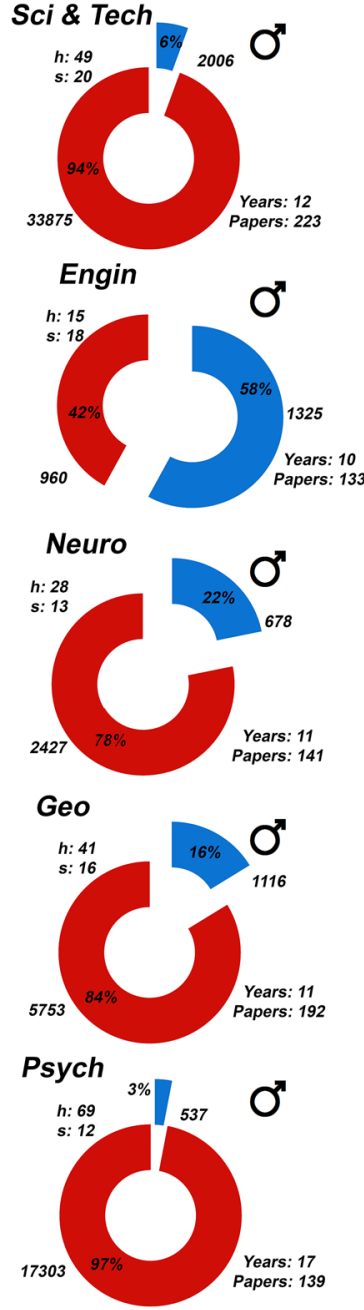
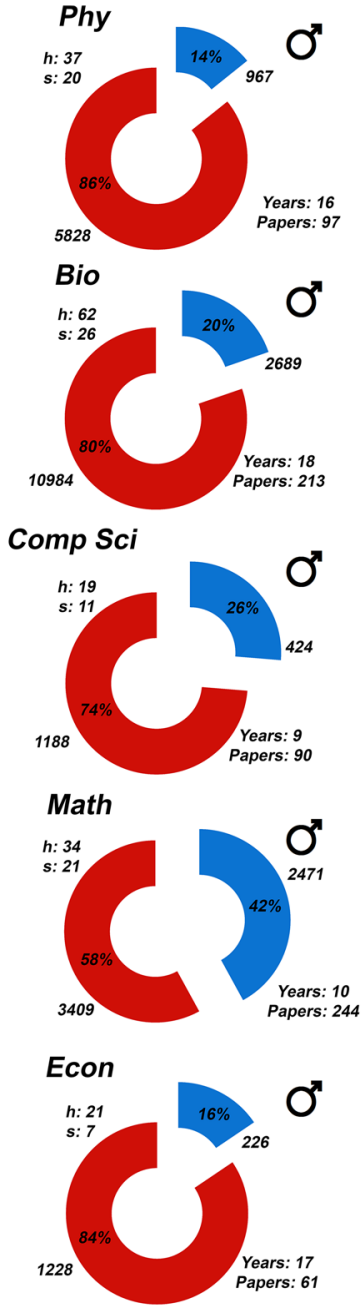
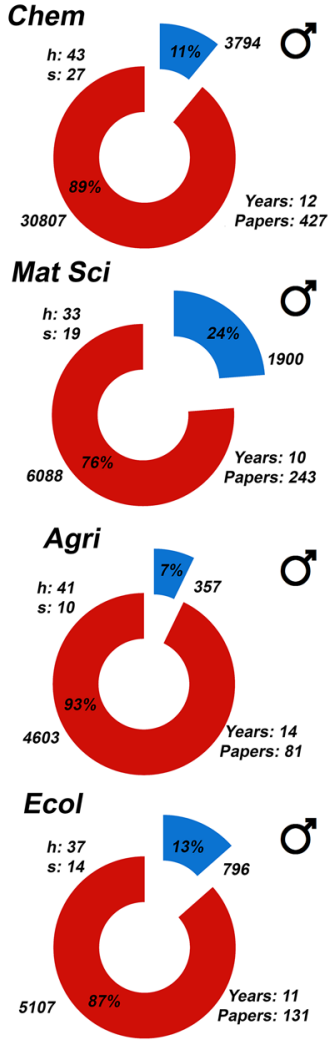

Mech

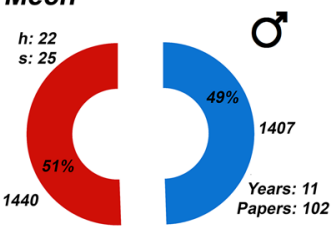

Fig. 3 Top $s$-index scorer per category. The highest scorers are depicted along with key information including category, gender (manually verified), $h$ (excluding self-citations), $s$, papers, years publishing, and proportion of citations that are self (self-citations in blue and nonself-citations in red). (Color figure online)

ratio of self-citations to total citations, which is hidden if you only consider the $s$-index by itself. Thus, it is important to keep track of all the relevant self-citation information and include it alongside nonself-citation data.

\section{Measuring the ratio of self/total citation}

Measuring the ratio of self to total citations for authors and each of their papers (see for example Fig. 1b) clearly shows the rate at which individuals build on their own ideas in relation to their peers, which is missing in current evaluation procedures. Furthermore, the 


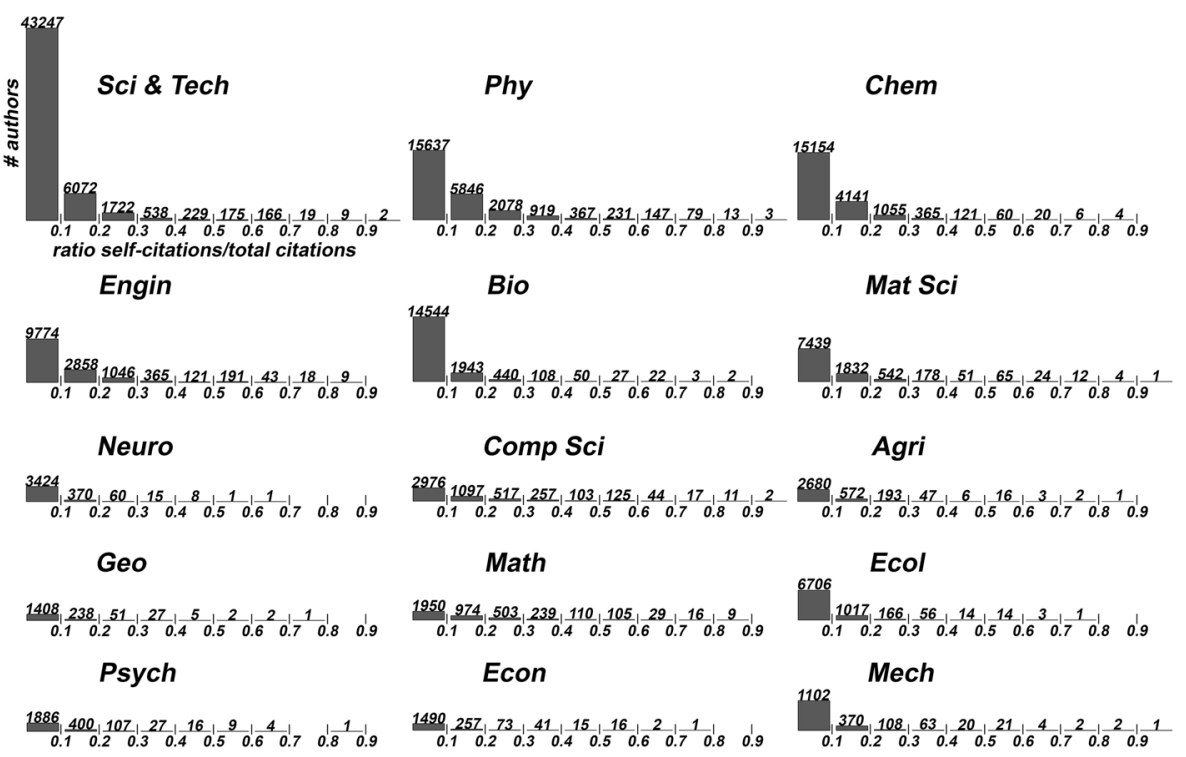

Fig. 4 Measuring the amount of self-citation in the total. Self/total citation ratios were calculated for the authors in each category. The exact amount of authors at a particular range of ratio is specified. For example, 15,637 researchers in category Phy have a ratio of self/total citations at or below 0.1

ratio can serve as an indicator for potential abuse of self-citation by flagging authors that self-cite disproportionately/excessively relative to the norm. In the current study $75 \%$ of the authors that self-cite have a ratio below 0.1 , meaning that less than $10 \%$ of their total citations are self-citations. To see how the measurements breakdown according to category, see Fig. 4. Interestingly, there are a total of 1822 authors in the dataset with ratios that exceed 0.5. Setting thresholds to define acceptable behavior will not explain how such high self/total citation ratios are achievable. Also, doing so will penalize certain situations where a high ratio is legitimate. Rather experts with suitable research backgrounds must help on a case-by-case basis to determine the various factors (e.g., career stage, level of productivity, research topic, citation mentality) that contribute to such high outcomes.

Figure 5 shows that the ratio of self/total citations tends to decrease as the $s$-index becomes larger, which we observed irrespective of category. Thus in most cases, authors with high $s$-scores are highly cited by their community of peers. This reinforces the idea that we should treat self-citation as a sign of progress (Mishra et al. 2018) rather than viewing the practice suspiciously, or worse scrapping the data altogether. As Cooke and Donaldson have argued, such an observation should be expected from researchers that have published year after year on focused topics with papers building on their own ideas and discoveries (Cooke and Donaldson 2014). It does not make sense to hide self-citation data when making bibliometric assessments. And importantly, when concerns arise over behavior, for example, when an author has a high ratio of self/total citations along with a high $s$-index, expert panels will have all the relevant data to aid in making sound judgments. 

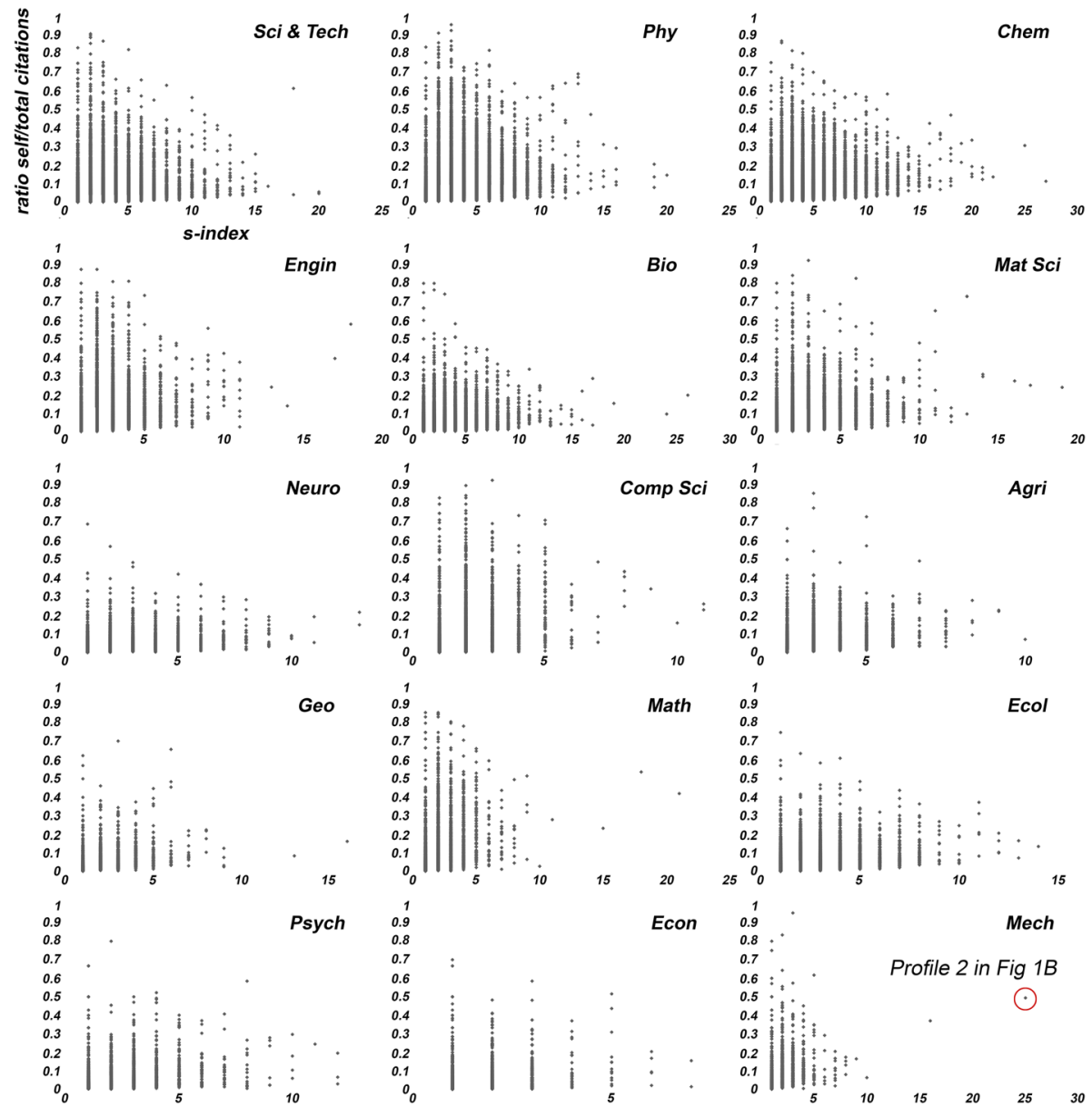

Fig. 5 The ratio of self/total citation as a function of increasing $s$-index

\section{Conclusion}

Clarifying citation buildup will help to accurately evaluate how the various author-related factors influence bibliometric footprint. The justification for doing this is that large-scale citation networks are heterogeneous and contain diverse citation mixing patterns that cannot be summarized using a single global measure (e.g., total citation counts, $h$-index). Instead of curation, we should adopt methods that utilize all the citation data, but in a way that carefully accounts for factors such as self-citation, collaboration, and "citation farms". Only then can we begin to fully appreciate authors' behavior and performance in relation to citation records. Towards this goal, we have shown here how to account for self-citation without introducing distortions. Future efforts centered on clarifying citations will better inform policymakers, funding agencies, hiring/promotion/award committees, and the general public about the value of published research. 


\section{Materials and methods}

We extracted authors' citation data from the 2016 Web of Science (WoS) database produced by Clarivate Analytics and hosted by the Leibniz Institute for Information Infrastructure (FIZ Karlsruhe). The database contains 50,040,717 records for a period of publishing from 1965 to 2016. Only authors possessing a unique identifier, either ORCID or ResearcherID, were included in the study. For sorting authors into specific research domains we utilized the WoS subject categorization scheme, where we define a given author's primary field of study as the category (e.g., Physics, Chemistry, Biology) containing the highest volume of publications. We analyzed categories if they contained at least 1000 unique authors. Citation queries were run using the Oracle SQL language.

Acknowledgements Open Access funding provided by Projekt DEAL. This work was funded by BMBF (Federal Ministry of Education and Research, Germany) under grant number 01PQ17001, the "Analyzing Self-citations in Web of Science (WoS)" project (http://bit.ly/Selfcitations-project), in the Competence Centre for Bibliometrics. A preprint version of this manuscript (Kacem et al. 2019) is available at bioRxiv https ://www.biorxiv.org/content/10.1101/2019.12.20.884031v1.

Author contributions AK: Formal analysis, investigation, methodology, validation, writing-reviewing and editing. JWF: conceptualization, formal analysis, funding acquisition, methodology, writing —original draft, writing — reviewing and editing. PM: roles: conceptualization, formal analysis, funding acquisition, methodology, project administration, resources, supervision, writing—reviewing and editing.

Open Access This article is licensed under a Creative Commons Attribution 4.0 International License, which permits use, sharing, adaptation, distribution and reproduction in any medium or format, as long as you give appropriate credit to the original author(s) and the source, provide a link to the Creative Commons licence, and indicate if changes were made. The images or other third party material in this article are included in the article's Creative Commons licence, unless indicated otherwise in a credit line to the material. If material is not included in the article's Creative Commons licence and your intended use is not permitted by statutory regulation or exceeds the permitted use, you will need to obtain permission directly from the copyright holder. To view a copy of this licence, visit http://creativecommons.org/licenses/by/4.0/.

\section{References}

Bartneck, C., \& Kokkelmans, S. (2011). Detecting h-index manipulation through self-citation analysis. Scientometrics, 87(1), 85-98. https://doi.org/10.1007/s11192-010-0306-5.

Cooke, S., \& Donaldson, M. (2014). Self-citation by researchers: Narcissism or an inevitable outcome of a cohesive and sustained research program? Ideas in Ecology and Evolution. https://doi.org/10.4033/ iee.2014.7.1.e.

Cousijn, H., Kenall, A., Ganley, E., Harrison, M., Kernohan, D., Lemberger, T., et al. (2018). A data citation roadmap for scientific publishers. Scientific Data, 20(5), 180259. https://doi.org/10.1038/sdata .2018 .259 .

Flatt, J. W., Blassime, A., \& Vayena, E. (2017). Improving the measurement of scientific success by reporting a self-citation index. Publications, 5, 20. https://doi.org/10.3390/publications5030020.

Fowler, J. H., \& Aksnes, D. W. (2007). Does self-citation pay? Scientometrics, 72(3), 427-437. https://doi. org/10.1007/s11192-007-1777-2.

Hicks, D., Wouters, P., Waltman, L., de Rijcke, S., \& Rafols, I. (2015). Bibliometrics: The Leiden Manifesto for research metrics. Nature, 520(7548), 429-431. https://doi.org/10.1038/520429a.

Hirsch, J. E. (2005). An index to quantify an individual's scientific research output. Proceedings of the National Academy of Sciences, 102(46), 16569-16572. https://doi.org/10.1073/pnas.0507655102.

Ioannidis, J. P. A., Baas, J., Klavans, R., \& Boyack, K. W. (2019). A standardized citation metrics author database annotated for scientific field. PLoS Biology, 17(8), e3000384. https://doi.org/10.1371/journ al.pbio.3000384.

Kacem, A., Flatt, J. W., \& Mayr, P. (2019). Tracking self-citations in academic publishing. https://doi. org/10.1101/2019.12.20.884031. 
Mishra, S., Fegley, B. D., Diesner, J., \& Torvik, V. I. (2018). Self-citation is the hallmark of productive authors, of any gender. PLoS ONE, 13(9), 0195773.

Seeber, M., Cattaneo, M., Meoli, M., \& Malighetti, P. (2019). Self-citations as strategic response to the use of metrics for career decisions. Research Policy, 48(2), 478-491. https://doi.org/10.1371/journ al.pone.0195773.

Zhivotovsky, L. A., \& Krutovsky, K. V. (2008). Self-citation can inflate h-index. Scientometrics, 77(2), 373375. https://doi.org/10.1007/s11192-006-1716-2. 